\title{
A Network Pharmacology Study to Explore the Underlying Mechanism of Safflower (Carthamus Tinctorius $L$.) in the Treatment of Coronary Heart Disease
}

Qibing Liu ( $\sim$ qibing.liu@hainmc.edu.cn )

Hainan Medical University

Qingwen Meng

The First Affiliated Hospital of Hainan Medical University

Huajiang Liu

The First Affiliated Hospital of Hainan Medical University

Haolin Wu

Hainan Medical University

shun Ding

The First Affiliated Hospital of Hainan Medical University

Chaoling Tang

The First Affiliated Hospital of Hainan Medical University

\section{Xinyin Fu}

The First Affiliated Hospital of Hainan Medical University

\section{Xingyue Fang}

The First Affiliated Hospital of Hainan Medical University

\section{Yiqian $\mathrm{Xu}$}

Hainan Medical University

\section{Bocen Chen}

Hainan Medical University

\section{Yiqiang Xie}

Hainan Medical University

\section{Research Article}

Keywords: safflower, coronary heart disease, network pharmacology, SIRT1/Fox01 signaling pathway

Posted Date: January 18th, 2022

DOI: https://doi.org/10.21203/rs.3.rs-1176341/v1 
License: (c) (i) This work is licensed under a Creative Commons Attribution 4.0 International License. Read Full License 


\section{Abstract}

Background: Safflower has long been used to treat coronary heart disease (CHD). However, the underlying mechanism remains unclear. The goal of this study was to predict the therapeutic effect of safflower against $\mathrm{CHD}$ using a network pharmacology, and to explore the underlying pharmacological mechanisms in vitro.

Methods: Firstly, we obtained relative compounds of safflower based on the TCMSP database. The TCMSP and PubChem databases were used to predict targets of these active compounds. Then we built CHD target database by DisGeNET database. The protein-protein interaction (PPI) network graph of overlapping genes was obtained after supplying the common targets of safflower and CHD into the STRING database. The PPI network was then used to determine the top ten most significant hub genes. Furthermore, DAVID database was utilized for the enrichment analysis on Gene Ontology (GO) and Kyoto Encyclopedia of Genes and Genomes (KEGG). To validate these results, a cell model of CHD was established in EAhy926 cells using oxidized low-density lipoprotein (ox-LDL).

Results: Safflower was determined to have 189 active compounds. The TCMSP and PubChem databases were used to predict 573 targets of these active compounds. The DisGeNET database was used to identify 1576 genes involved in the progression of CHD. The top ten hub genes were $A L B, I L 6, I L 1 B$, VEGFA, STAT3, MMP9, TLR4, CCL2, CXCL8, and IL 10. GO functional enrichment analysis yielded 92 entries for biological process (BP), 47 entries for cellular component (CC), 31 entries for molecular function (MF), and 20 signaling pathways were obtained from KEGG pathway enrichment screening. Based on these findings, the FoxO signaling pathway is critical in the treatment of CHD by safflower. The vitro Results showed that safflower had an ameliorating effect on ox-LDL induced apoptosis and mitochondrial membrane potential. Western blotting results showed that compared to the model group, safflower decreased Bax expression and acetylation of FoxO1 proteins while increasing the expression of Bcl-2 and SIRT1 proteins.

Conclusions: Safflower targets multiple pathways during CHD treatment, and can exert anti-apoptotic effects by regulating the expression of $\mathrm{BAX}, \mathrm{BCL}-2$, and SIRT1/FoxO1 signaling pathway-related proteins.

\section{Background}

According to the American Heart Association's Heart Disease and Stroke Statistics (2020 Edition), cardiovascular disease is a growing public health concern worldwide [1]. According to the China Cardiovascular Health and Disease Report 2020 Edition, the prevalence of cardiovascular disease in China is on the rise, with an estimated 330 million people suffering from the disease. Cardiovascular disease is the primary cause of death in both urban and rural areas. As the primary risk factor for cardiovascular death, coronary heart disease (CHD) has long caused serious harm to families and society. Interventional therapy and medicine are the main treatment strategies for patients with CHD. 
Although these methods are rapid and effective, they are also accompanied by side effects, such as postoperative rebleeding and adverse reactions.

Traditional Chinese medicine (TCM) is multi-targeted and multi-directional, and is therefore used in the treatment of CHD. Dehydrated flowers of the safflower plant (Carthamus tinctorius L.), which belongs to the subclass Dicotyledoneae and family Compositae, are commonly used in clinics to treat CHD, hypertension, and cerebrovascular diseases, as well as to enhance blood flow and remove blood blockage[2]. Modern pharmacological studies have shown that safflower has vasodilating, anticoagulant, blood pressure-lowering, antioxidant, anti-inflammatory, and analgesic effects[3]. Previous studies have demonstrated that safflower inhibits the JNK1/2-NF-KB pathway, which prevents lipopolysaccharide (LPS)-induced TNF-signaling activation and cell death in H9c2 cardiomyoblast cells[4]. However, it is difficult to determine the mechanism of safflower in the treatment of CHD; the active target mechanism of its effective chemical components particularly require further exploration.

The network pharmacology approach is based on the biochemical construction of a drug-componentdisease target network. It explores the relationship between the target and pathway, while clarifying the molecular mechanism. This technique delineates the holistic and systematic features of TCM, and it has significant benefits over traditional approaches in terms of comprehending and depicting comprehensive mechanisms. It is an approach used to discover active components, validate the efficacy and mechanisms of drug actions and interactions, confirm the beneficial active components of the metabolic process, and investigate prototype compounds. It has recently been used to demonstrate potential mechanisms of TCM therapy for illnesses such as CHD and diabetes. By creating a network model, network pharmacology may be utilized to understand the links between components and pathways.

In the current study, the potential mechanism of safflower in CHD therapy was evaluated using a networkbased systematic analysis. A comparative analysis was also performed to identify overlapping genes. Furthermore, overlapping genes and hub nodes were used to build protein-protein interaction (PPI) and module networks, which were then analyzed for Gene Ontology (GO) and Kyoto Encyclopedia of Genes and Genomes (KEGG) pathway enrichment. We aimed to investigate the potential therapeutic targets of safflower in the treatment of CHD, which would serve as a foundation for further research into the pharmacological mechanism of safflower. Subsequently, we used in vitro cellular experiments to validate the predicted molecular mechanism of safflower in coronary heart disease therapy as determined by network pharmacology. The results of these experiments provide a foundation for the clinical application of safflower in CHD patients. The research process is illustrated in Figure1.

\section{Methods}

\section{Network Pharmacology}

\section{Construction of the effective compounds of safflower}


The effective compounds of safflower were obtained by screening the Traditional Chinese Medicine Systems Pharmacology Platform (TCMSP) database (http://lsp.nwu.edu.cn/tcmsp.php). TCMSP is an open database that contains the oral bioavailability (OB) of nearly 500 Chinese herbal medicines and their drug similarity (DL), blood-brain barrier (BBB), intestinal epithelial pass permeability (Caco-2), and other reference values. The compounds contained in safflower were screened using the TCMSP database, with oral bioavailability set to $\mathrm{OB} \geq 30 \%$ and drug-likeness set to $\mathrm{DL} \geq 0.18$.

\section{Screening for the targets of safflower}

Typically, medicinal components interact with relevant biological pathways via their targets. To estimate the targets of compounds found in safflower, details on the molecular structure of the bioactive constituents in safflower were obtained from the TCMSP and PubChem databases

(https://pubchem.ncbi.nlm.nih.gov/). The top 100 target genes corresponding to each compound were searched from the chemical-gene co-occurrences in the literature module of the PubChem database. Subsequently, genes corresponding to the targets of the safflower compounds were determined using the STRING database (https://www.string-db.org/).

\section{Collection of targets related to the pathogenesis of CHD}

Using the keyword "coronary heart disease," target genes were located in DisGeNet (https://www.disgenet.org/). DisGeNET is an exploratory platform containing genes associated with human diseases and their comorbidities. According to Score_gda $>0.1$, targets related to the pathogenesis of $\mathrm{CHD}$ were selected.

\section{Identification of common targets of CHD and safflower}

A Venn diagram depicting the intersection of safflower targets and CHD targets was constructed using Venny 2.1 (BioinfoGP, Madrid, Spain). The intersecting genes were discovered by comparing safflower target genes to CHD-related pathogenesis targets.

\section{Disease-Compound-Target-Pathway Network construction of safflower in the treatment of CHD}

The Cytoscape software (version: 3.6.0) was used to construct the disease-compound-target-pathway network, followed by network topology analysis. Cytoscape is a visualization software that can integrate biomolecular interaction networks, data on high-throughput gene expression, and other molecular details. 


\section{Construction and analysis of protein-protein interaction (PPI) network diagram}

To explain the relationship between projected targets and other human proteins, a PPI network was created, which has been shown to be a viable technique for drug development. The protein-protein interaction network of potential therapeutic targets of safflower for CHD was built using the STRING database. In the designated setting, protein associations with a confidence score greater than 0.4 were selected after duplicates were removed.

\section{Examination of the hub gene}

The PPI network was imported into Cytoscape using the CytoHubba plugin and the MCC algorithm was used. The top ten hub genes targeted by safflower to treat $\mathrm{CHD}$ were identified.

\section{Analyses of pathway enrichment for core targets}

The screened targets were analyzed using the DAVID (https://david.ncifcrf.gov/) database for GO bioprocess analysis and KEGG pathway analysis. GO enrichment analysis comprises the following three aspects: biological process (BP), molecular function (MF), and cellular component (CC). The GO enrichment analysis results were selected based on P-values, and the top 10 pathways were selected. KEGG can be used to evaluate the target signaling pathways of medicines and identify those with the highest correlation, which is critical for determining the potential mechanism of safflower in CHD therapies. Finally, the threshold value of $P<0.05$ was determined. Major pathways were identified, and bioinformatics software was used to map the signaling pathways. The top 20 pathways were selected based on the outcomes of KEGG pathway enrichment analysis.

\section{Experimental validation}

\section{Cell culture and treatments}

EAhy 926 cells were purchased from the Cell Source Center, Chinese Academy of Sciences (Shanghai, China). Cells were commonly cultivated in Dulbecco's modified Eagle's medium (DMEM), $10 \%$ fetal bovine serum (FBS), and 1\% penicillin/streptomycin. The cells were randomly separated into three groups during the logarithmic growth phase: the control group, oxidized low-density lipoprotein (ox-LDL) group (obtained from Yeasen Biotechnology, Shanghai, China), and safflower group. Each group had at least three replicates, and cell counting was performed to ensure that the number of cells in each group was the same. Except for the control group, the cells were cocultured with $100 \mu \mathrm{g} / \mathrm{ml}$ ox-LDL for $24 \mathrm{~h}$ and then 
switched to normal medium or safflower drug-containing medium for another $24 \mathrm{~h}$, then collected for further analysis.

\section{Preparation of drug-containing serum}

Ten male Sprague-Dawley (SD) rats ( $180 \pm 20 \mathrm{~g}, 4$ weeks old) were provided by Changsha Tianqin Biotechnology Co. Ltd. (Changsha, China). All SD rats were housed under conventional conditions (indoor temperature $24 \pm 2^{\circ} \mathrm{C}$, humidity $60 \pm 15 \%, 12 \mathrm{~h} / 12 \mathrm{~h}$ light/dark cycles) and provided with equal access to standard rodent feed and water. All experiments were conducted in accordance with China's National Guidelines for Experimental Animal Care and Use.The study was approved by the Ethical Committee of the First Affiliated Hospital of Hainan Medical University (Haikou, China). SD rats were categorized into two groups for the preparation of safflower drug-containing serum $(n=5)$ and blank serum $(n=5)$. The typical human dose of safflower $(10 \mathrm{~g})$ was converted to an appropriate drug dosage for the SD male rats according to body surface area. SD rats were given a safflower solution $(1 \mathrm{~g} / \mathrm{kg})$ intragastrically via gavage for 7 days. The normal control group were given an equal volume of normal saline (NS). Blood samples were taken from the aorta one hour after the last gavage, and all rats were anesthetized with an intraperitoneal injection of chloral hydrate $(350 \mathrm{mg} / \mathrm{kg})$ before being sacrificed by via exposure to $\mathrm{CO}_{2}$. The supernatant was extracted, filtered, and inactivated in a water bath at $56^{\circ} \mathrm{C}$ for $30 \mathrm{~min}$, and the sera from the same group was mixed and stored at $-20^{\circ} \mathrm{C}$.

\section{Cell viability measurements}

A Cell Counting Kit-8 was used to check the viability of cells (CCK8; Dojindo Laboratories, Kumamoto, Japan). When cells reached $80 \%$ confluence, they were treated with ox-LDL at $0,25,50,100$, or $200 \mu \mathrm{g} / \mathrm{ml}$ for $24 \mathrm{~h}$. Subsequently, CCK8 solution $\left(10 \mu \mathrm{l} / 100 \mu \mathrm{l}\right.$ medium) was added to the media for $2 \mathrm{~h}$ at $37^{\circ} \mathrm{C}$ in a 96-well plate. A microplate reader (EPOCH, BioTek, Winooski, VT, USA) was used to measure the absorbance of the media at $450 \mathrm{~nm}$.

\section{TUNEL staining}

TUNEL staining is a technique used to detect DNA fragments that have formed during apoptosis (Abcam, Cambridge, UK). In each group, apoptotic cells were counted in three random fields using a fluorescent microscope.

\section{Detection of mitochondrial membrane potential by JC-1}

A JC-1 fluorescent probe was used to measure mitochondrial membrane potential according to the manufacturer's instructions (Beyotime Biotechnology, Shanghai, China). EAhy926 cells were incubated 
with $1 \mathrm{ml} \mathrm{JC}-1$ at $37^{\circ} \mathrm{C}$ for 20 min, washed twice with PBS, then observed and photographed using a fluorescent microscope.

\section{Western blot}

Cells were collected and lysed on ice in 6-well plates. BCA protein assay kits were used to measure the concentrations (Thermo Fisher Scientific, Waltham, MA, USA). SDS-PAGE was used to separate the protein samples, which were then transferred to polyvinylidene fluoride (PVDF) membranes. After blocking for $2 \mathrm{~h}$ at room temperature with $5 \%$ nonfat milk in Tris-buffered solution containing $0.1 \%$ Tween 20 (TBST), membranes were incubated overnight at $4{ }^{\circ} \mathrm{C}$ with primary antibodies (1:1000) against Bax (Abcam), Bcl-2 (Abcam), Sirt1 (Thermo Fisher Scientific), acetylated Fox01 (Thermo Fisher Scientific), and $\beta$-actin (Abcam). After three washes, the luminescence developed, and the gel imager was exposed for imaging. The strips were analyzed for grayscale using Image $J$ software (National Institutes of Health, Bethesda, MD, USA) and processed for semi-quantitative analysis, with three replicates each.

\section{Statistical analysis}

All experimental data are presented as mean \pm SD. Prism software was used for data processing and analysis, and one-way analysis of variance was used for comparison between groups. Significance was set to $P<0.05$.

\section{Results}

\section{Screening for safflower active compounds}

We obtained 189 safflower compounds from the TCMSP database. Using the screening conditions of $\mathrm{OB} \geq 30 \%$ and $\mathrm{DL} \geq 0.18,34$ active compounds that met the criteria were obtained, including 6hydroxykaempferol, baicalein, qt_carthamone, quercetagetin, beta-carotene, baicalin, beta-sitosterol, stigmasterol, kaempferol, and quercetin. Precise information on the main bioactive components is shown in Table 1.

\section{Safflower for the treatment of CHD targets}

The TCMSP and PubChem databases were used to retrieve 2527 targets from the 34 candidate components. After expelling the overlapping targets, a total of 573 targets of safflower compounds were acquired. The DisGeNET data comprises 1576 genes associated with CHD. 


\section{Overlapping genes between safflower and CHD molecular target genes}

Using the web tool Venny 2.1, the intersection of the targets corresponding to CHD and safflower active compounds was determined using a Venn diagram. According to the findings, a total of 239 putative targets related to both CHD and safflower were identified (Figure 2).

\section{PPI Network Analysis}

The STRING database was used to create a PPI network to further elucidate the biological mechanisms underlying the pharmacological effects of safflower on CHD. The nodes and edges in the network indicate proteins and protein-protein interactions, respectively. According to the results, the network had 239 nodes and 5819 interactions (Figure 3). The average node degree was 48.7, with a local clustering coefficient of 0.634 .

\section{Hub gene scanning}

The hub gene was screened in the interaction network using CytoHubba, a Cytoscape plug-in. The top 10 hub genes for safflower treatment of CHD were identified using the MCC algorithm (Figure 4), and a hub gene network diagram was created. The MCC algorithm was used to assess the top ten safflower hub gene networks for treating CHD, with the colors red and yellow indicating the network's significance.

\section{Safflower functional enrichment assessment in CHD}

The BP, CC, and MF annotations of the 239 selected proteins were determined using the DAVID database. $\mathrm{GO}$ analyses revealed 92 enriched $\mathrm{GO}$ terms in the category of biological process (BP), including cholesterol homeostasis, lipoprotein metabolic process, high-density lipoprotein particle remodeling, cholesterol efflux, and reverse cholesterol transport. GO analyses indicated 47 enriched GO terms in the category of cellular component (CC), including extracellular space, extracellular region, cell surface, and receptor complex. GO analyses reflected 31 enriched GO terms in the molecular function (MF) category, including heme binding, cholesterol transporter activity, transcription factor binding, RNA polymerase II transcription factor activity, and ligand-activated sequence-specific DNA binding (Figure 5).

\section{KEGG pathway enrichment explanation}

The 239 putative targets were registered into the DAVID database for KEGG pathway enrichment analysis to explain the crucial signaling pathways targeted by safflower in $\mathrm{CHD}$ therapeutic approaches. Figure 6 depicts the KEGG pathway enrichment analysis. The top 20 pathways were tested using a combination of 
count parameters and $P$-values, along with the HIF-1 signaling pathway, PI3K-Akt signaling pathway, tolllike receptor signaling pathway, FoxO signaling pathway, and TNF signaling pathway.

\section{Disease- Compound-Target-Pathway network}

To more specifically define the method of action of safflower in the treatment of CHD, a network of disease-compound-target-pathways was constructed. The network, shown in Figure 7, was constructed of disease, compound, protein targets, and pathways, with 288 nodes and 1245 edges. As for CHD, 27 components communicated with 239 target proteins and were linked to 21 pathways. Based on these findings, the FoxO signaling pathway was defined as one of the most valuable signaling pathways during the treatment of $\mathrm{CHD}$ with safflower. Figure 8 illustrates the targets for the signaling pathways that were obtained from the KEGG database (http://www.kegg.jp/kegg/mapper.html).

\section{Ox-LDL decreased the cell viability of EAhy926 cells}

Different concentrations of ox-LDL $(0,25,50,100$, and $200 \mu \mathrm{g} / \mathrm{ml})$ were applied to EAhy 926 cells for $24 \mathrm{~h}$. The survival rates of each group of cells are shown in Figure 9. When the concentration of ox-LDL exceeded $50 \mu \mathrm{g} / \mathrm{ml}$ for $24 \mathrm{~h}$, cell viability decreased. At $100 \mu \mathrm{g} / \mathrm{ml}$, ox-LDL reduced cell viability by approximately $50 \%$. According to the experimental results, ox-LDL $100 \mu \mathrm{g} / \mathrm{ml}$ applied for $24 \mathrm{~h}$ could inhibit EAhy 926 cell viability more effectively, which was used as a modeling condition for subsequent experiments.

\section{Safflower attenuated ox-LDL induced apoptosis of EAhy926 cells}

The results of apoptosis detection by TUNEL are shown in Figure 10, and different numbers of apoptotic cells were observed in the normal, model, and safflower groups. The highest number of apoptotic cells was observed in the model group, and the apoptosis of EAhy 926 cells was reduced after safflower treatment compared with the previous treatment.

\section{Effects of Safflower on the mitochondrial membrane potential of ox-LDL induced EAhy926 cells}

Compared to the control group, the cells in the model group had higher green and lower red fluorescence, and their mitochondrial membrane potential was significantly lower. After safflower intervention, the green fluorescence in EAhy 926 cells gradually decreased, and the red fluorescence gradually increased compared to the control group (Figure 11). The differences were statistically significant. According to 
these findings, safflower may effectively resist the ox-LDL-induced decrease in mitochondrial membrane potential in EAhy926 cells.

\section{Effect of safflower on ox-LDL induced apoptosis by modulating the SIRT1/FoxO signaling pathway}

The FoxO signaling pathway, which was ranked in the top 20 in the KEGG pathway analysis, plays a crucial role, and the SIRT1 protein is part of the upstream signaling pathway. SIRT1 has a deacetylating effect and is inhibited under physiological conditions; however, SIRT1 activation, increased expression, and deacetylation activity are enhanced when cells are stimulated by pathological factors such as ischemia, hypoxia, and LPS. Activated Sirt1 can deacetylate Fox01, and deacetylated Fox01 enters the cell plasma from the nucleus. FoxO1 exits the nucleus and loses its role in inhibiting Bax and promoting $\mathrm{Bcl}-2$ expression, resulting in increased Bax expression, decreased $\mathrm{Bcl}-2$ expression, and apoptosis.

We therefore tested the protein expression of SIRT1 and acetylation of Fox01 (Ac-Fox01) in EAhy926 cells to determine how safflower affected ox-LDL-induced cardiac damage. Compared to control cells, the protein levels of SIRT1 were considerably reduced in ox-LDL treated cells, and the protein levels of AcFox01 were significantly increased in ox-LDL-treated cells, as shown in Fig. 12. However, pretreatment with safflower increased the expression of SIRT1 and simultaneously decreased the expression of AcFox01 compared to the ox-LDL group.

As demonstrated in Fig. 12, ox-LDL significantly increased relative levels of Bax protein and significantly lowered relative levels of $\mathrm{Bcl}-2$ protein, which was significantly inhibited by safflower pretreatment. These results indicate that the mechanism of safflower inhibition of ox-LDL-induced apoptosis in EAhy 926 cells is associated with its role in regulating the SIRT1/FoxO1 pathway.

\section{Discussion}

$\mathrm{CHD}$ is a severe hazard to human health because it is a complex cardiovascular disease. It is characterized by ischemia, hypoxia, or necrosis of the myocardium caused by narrowing or blockage of the heart vessels due to coronary artery sclerosis or spasm. CHD is caused by a variety of factors. Single target drugs are typically ineffective for the treatment of this multifactorial disease. Furthermore, repeated or continuous high-dose administration of a single specific drug can result in drug resistance. As a result, the development of new multitarget drugs is critical. TCM has multi-component and multi-target qualities, meaning it can target multiple biological systems to manage symptoms and treat basic problems.

Safflower has been used to treat CHD for over a thousand years[5-7], but its bioactive components and processes are unknown, limiting its therapeutic applicability. To address this difficulty, we used a network pharmacology approach. The active substances and targets of TCM can be predicted more accurately using network pharmacology[8]. Many studies are currently being conducted to investigate the complexities of the components, targets, pathways, and mechanisms of action of herb pairings or herbal 
formulae using network pharmacology [9-11]. The pharmacologically active components and mechanisms involved in CHD treatment were predicted using the network pharmacology method in this study, and several proteins were verified via WB monitoring. The components of safflower with $\mathrm{OB} \geq 30 \%$ and $D L \geq 0.18$ were found to be pharmacokinetically active, meaning they are presumably absorbed and transported throughout the body. Accordingly, the network pharmacological study of safflower revealed 34 chemicals, including $\beta$-sitosterol, kaempferol, quercetin, baicalein, flavoxanthin, lignan, precarthamin, carthamone, quercetagetin, and 3-methoxydaidzein, that have the potential to treat CHD. $\beta$-sitosterol protects the heart by modulating antioxidant enzymes via the estrogen/phosphatidylinositol 3-kinase pathway[12]. In vitro, TNF-induced apoptosis and inflammation in human umbilical vein endothelial cells are suppressed by quercetin via the NF-KB and AP-1 signaling pathways[13]. Kaempferol reduces ox-LDLinduced apoptosis in human endothelial cells by increasing autophagy and inhibiting the $\mathrm{PI3K} / \mathrm{Akt} / \mathrm{mTOR}$ pathway[14]. Kaempferol's positive effects in postmenopausal AS are linked to the $\mathrm{PI3K} / \mathrm{AKT} / \mathrm{Nrf} 2$ pathways, which are influenced by $\mathrm{G}$ protein-coupled estrogen receptor activation[15]. Based on these findings, baicalein has anti-inflammatory properties, most likely by activating the AMPK/Mfn-2 axis and inhibiting downstream MAPK/NF-KB signaling[16]. Baicalin may prevent atherosclerosis by increasing cholesterol efflux from macrophages and delaying the production of foam cells via the PPAR-LXR-ABCA1/ABCG1 pathway[17]. According to research, baicalein, a 12/15-LOX inhibitor, can prevent myocardial I/R injury through a variety of mechanisms, and it could be used to treat acute myocardial infarction [18].

Because of the high degree of node and closeness centrality in this network, VEGFA, STAT3, IL-6, IL-1B, MMP9, CCL2, CXCL8, TLR4, IL-10, and ALB were chosen as candidate core genes. VEGFA is a direct target gene of miR-451 according to TargetScan and dual-luciferase reporter gene assay data, and could be a new biomarker for CHD[19]. In a PDGF-BB-stimulated atherosclerosis model, the researchers discovered that circLMF1 deficiency was found to suppress cell survival, cell cycle progression, and migration, possibly via the miR-125a-3p/VEGFA or FGF1 axis[20]. In endothelial cells, fatty acid-binding protein 4 serves as a hub for angiogenic and metabolic signaling pathways. FABP4 is activated by VEGFA in endothelial cells, and inhibiting FABP4 prevents the effects of VEGFA[21]. In the PDGF-BB-stimulated atherosclerosis model it was discovered that circLMF1 deficiency inhibited cell survival, cell cycle progression, and migration, possibly via the miR-125a-3p/VEGFA or FGF1 axis[22]. Based on its translocation, STAT3 can be classified as nuclear or mitochondrial, and both are thought to play essential roles in the development of atherosclerosis, including endothelial cell malfunction, macrophage polarization, inflammation, and immunology[23-26]. The JAK2/STAT3 pathway is intimately linked to the IL-6 cytokine family, which plays a critical role in endothelial cell dysfunction during atherosclerosis[27]. Atherosclerosis is caused by the migration of vascular smooth muscle cells (VSMCs), and matrix metalloproteinases (MMPs) play important roles in VSMC migration[28]. MMP9 is a significant regulator of the inflammatory response. The inflammatory response recruits leukocytes and produces a variety of cytokines and chemokines that increase MMP9 release, which also promotes the activation of inflammatory factors such as IL-1, resulting in a positive feedback loop[29]. When AS strikes, MMP9 levels rise, and the MMP9/NGAL complex level is closely linked to plaque vulnerability[30]. 
CCL2 was found in small amounts in normal arteries but was more commonly found in the intima. CCL2 and the expression of related receptors were significantly increased in diseased arteries, with relative differences between artery layers[31]. Interleukin-8 (IL-8, CXCL8) is an inflammatory agent that has been linked to the progression of atherosclerosis[32]. TLR4 is well known for its role as an important mediator of the innate immune response and has been linked to atherosclerosis initiation, progression, and plaque destabilization[33,34]. IL-10 is a pleiotropic cytokine that has been proposed as a hazard modifier for atherosclerosis[35]. One study showed that in response to inflammatory stimuli, IncRNA-CCL2 and CCL2 displayed coordinated upregulation, and their expression was associated with unstable symptomatic human atherosclerotic plaques[36]. The relationship between hypoalbuminemia, systemic albumin leakage, and soluble markers of systemic inflammation and endothelial injury has been studied in peritoneal dialysis patients, with results indicating that hypoalbuminemia is more frequently associated with inflammation and atherosclerotic disease, but little is known about the use of albumin in patients with coronary artery disease[37].

These targets are mostly connected with signal transduction pathways such as TNF, HIF-1, FoxO, Toll-like receptor, and PI3K-Akt signaling, according to our KEGG pathway analysis. As a pro-atherogenic cytokine, TNF increases the expression of cytokines and adhesion molecules, as well as the migration and mitogenesis of VSMCs and endothelial cells[38]. HIF-1 is a heterodimeric protein that belongs to the basic helix-loop-helix family and plays a major role in regulating cellular responses in low-oxygen environments. It acts on endothelial cells, vascular smooth muscle cells, and macrophages, and through cell-specific responses, it plays an essential role in the advancement of atherosclerosis[38]. Hypoxia increases macrophage glycolytic flux and upregulates proinflammatory activity in a manner that is dependent on both hypoxia-inducible factor -1 and 6-phosphofructo-2-kinase[39]. Multiple cell types and pathway cascades linked to atherosclerosis are affected by the PI3K-Akt signaling pathway. In atherosclerotic animal models, activation of this system via vascular endothelial dysfunction lowers the formation of atherosclerotic plaques[40,41]. TNF-induced endothelial cell inflammation is mediated by METTL14. The m6A alteration of Fox01, an essential transcription factor, was dramatically enhanced during endothelial inflammation[42]. Fox01, IL-6, TNF-a, miR-27a, and miR-23a expression levels were shown to be substantially linked to the severity of stenosis in the PBMCs of patients with CAD. Compared to healthy controls, the expression of SIRT1 was shown to be significantly lower in CAD patients[43]. Macrophage polarization plays an essential role in atherosclerosis, and M1 polarization and inflammation are generated by PAR2 activation via the F0X01-dependent pathway[44].

Accordingly, based on network pharmacology predictions, we mainly concentrated on the SIRT1/ACF0X01 signaling pathway for further exploration of the preventive role of safflower on endothelial cells. The $\mathrm{O}$ subfamily of Fox01 is a critical regulator of cell metabolism in a variety of organs, including the heart, where it plays a role in cardiac glucose and lipid metabolic pathways, as well as other metabolic pathways[45]. SIRT1 is a histone deacetylase that is stimulated by nicotinamide adenine dinucleotide $(\mathrm{NAD}+)$ and is valuable in energy metabolism. SIRT1 promotes endothelial angiogenesis, homeostasis, and remodeling by regulating Fox01 through its deacetylase activity[46]. The SIRT1/Fox01 signaling pathway plays a major role in oxidative stress, cell pathophysiological processes such as apoptosis, and 
metabolic regulation. SIRT1-induced deacetylation of Fox01 suppresses apoptosis and oxidative stress by upregulating MnSOD, Bcl-2, and $\mathrm{Bcl}-\mathrm{XL}$ and downregulating Bim and FasL[47]. FoxO1 is highly expressed in atherosclerotic plaques and appears to have an atheroprotective effect since Fox01 knockdown in endothelial cells in a mouse model reduced atherosclerosis[48]. SIRT1 deficiency enhances oxidative stress, inflammation, foam cell production, and atherosclerosis progression in endothelial cells[49]. SIRT1/Fox01 could be a promising target for preventing AS and arterial thrombosis[50]. In this study, SIRT1 expression increased while acely-Fox01 expression decreased in the model group, and SIRT1 expression decreased, while acely-Fox01 expression increased after safflower intervention.

Numerous studies have found that apoptosis is important for the pathogenesis of CHD[51]. After ox-LDL stimulation, the viability of EAhy 926 cells was significantly lowered, while the apoptosis rate increased, implying that ox-LDL can result in cell death. The viability of EAhy 926 cells was upregulated and the apoptosis rate was significantly lowered after safflower intervention, indicating that safflower inhibits oxLDL-induced apoptosis in endothelial cells, and inhibition of apoptosis may be the molecular mechanism by which safflower exerts its endothelial protective effect. BCL-2 and BAX are localized in the mitochondrial membrane and are involved in the permeability of the mitochondrial membrane to cytochrome $\mathrm{C}$. The homodimer formed by Bax is a channel for cytochrome $\mathrm{C}$ to enter the cytoplasm from the mitochondria and promotes the release of cytochrome $\mathrm{C}$. The heterodimer formed by $\mathrm{Bcl}-2$ and $\mathrm{Bax}$ can antagonize the function of Bax and inhibit the release of cytochrome $\mathrm{C}$. Cytochrome $\mathrm{C}$, which enters the cytoplasm from the mitochondria, activates caspase- 9 and eventually activates caspase- 3 through a series of downstream cascade reactions, and the activated caspase-3 mediates apoptosis[52]. Under oxLDL stimulation, Bcl-2 expression declined, Bax expression increased, and the Bcl-2/Bax ratio was significantly reduced in EAhy 926 cells. In addition, TUNEL staining showed an increase in the number of TUNEL-positive cells in ox-LDL-stimulated EAhy 926 cells, indicating that ox-LDL induced the apoptotic process in these cells. Meanwhile, the results of this study showed that safflower significantly inhibited the decrease in Bax expression, increase in Bcl-2 expression, and decrease in the Bax/Bcl-2 ratio in oxLDL-stimulated EAhy926 cells, and the positive TUNEL staining in ox-LDL-stimulated EAhy 926 cells was significantly reduced compared with the model group. It has been suggested that safflower has an inhibitory effect on ox-LDL-induced mitochondrial pathway apoptosis in endothelial cells, and antagonism of mitochondrial pathway apoptosis may be the molecular mechanism by which safflower exerts its protective effect on endothelial function.

The mitochondrial apoptotic pathway in endothelial cells is regulated by several signaling pathways, among which the SIRT1/Fox01 pathway can sense energy changes and regulate the expression of mitochondrial pathway apoptotic genes such as Bcl-2 and Bax. SIRT1 is deacetylated and is inhibited under physiological conditions; however, when cells are stimulated by pathological factors such as ischemia and hypoxia and LPS, SIRT1 is activated, resulting in increased expression and deacetylation activity. Activated SIRT1 can deacetylate Fox01, and the deacetylated Fox01 enters the cytoplasm from the nucleus[53, 54]; Fox01 leaves the nucleus and loses its role in inhibiting Bax and promoting Bcl-2 expression, resulting in increased Bax expression and decreased Bcl-2 expression, which ultimately leads to apoptosis. 


\section{Conclusion}

Using network pharmacology, the targets, mechanism of action, and associated signaling pathways of safflower in the therapy of CHD were assessed in this study. It was demonstrated that the effective compounds of safflower in the treatment of CHD may be dipalmitin, flavoxanthin, lignan, precarthamin, carthamone, quercetagetin, $\beta$-sitosterol, kaempferol, quercetin, baicalein, and 3 '- methoxydaidzein.

Possible targets include VEGFA, STAT3, IL-6, IL-1B, MMP9, CCL2, CXCL8, TLR4, IL-10, ALB, and putative pharmacological activities of other bioactive components linked to antioxidative stress, anti-apoptosis, and anti-inflammation. In addition, coronary artery disease can lead to activation of the SIRT1/FoxO1 signaling pathway and activation of the apoptotic signaling pathway, and safflower can reverse this result.According to the findings, network pharmacology predictions could be useful in illustrating the molecular mechanisms of the Chinese herbal drug safflower in the treatment of CHD.

\section{Abbreviations}

CHD: coronary heart disease; TCMSP: the Traditional Chinese Medicine Systems Pharmacology Platform; GO: Gene Ontology; KEGG: Kyoto Encyclopedia of Genes and Genomes; ox-LDL: oxidized low-density lipoprotein; BP: biological process; CC: cellular component; MF: molecular function; LPS:

lipopolysaccharide; OB: oral bioavailability; DL: drug similarity; BBB: blood-brain barrier; Caco-2: intestinal epithelial pass permeability; PPI: protein-protein interaction.

\section{Declarations}

\section{Ethics approval and consent to participate}

The animal experiments involved in this experiment were approved by the Ethics Committee of the First Affiliated Hospital of Hainan Medical College (Haikou, China).

\section{Consent for publication}

Not applicable.

\section{Availability of data and material}

We have presented all our main data in the paper.

\section{Competing interests}


The authors declare that the research was conducted in the absence of any commercial or financial relationships that could be construed as a potential conflict of interest.

\section{Funding}

This study was supported by the National Natural Science Foundation of China (No.81960663), Youth Incubation Fund Project of Hainan Medical College (No. HYPY201912), Innovative Research Team Project of Natural Science Foundation of Hainan Province (No. 2019CXTD407), Natural Science Foundation of Hainan Province Youth Project (No. 821QN401).

\section{Authors' contributions}

All the authors had read and approved the final manuscript. The all authors have read and approved the manuscript. MQW designed the study and had written the article. LHJ, DS, TCL and FXY had completed the network pharmacology mapping section. WHL, FXY, XYQ and CBC helped to perform the experiments. $X Y Q$ and LQB revised the article.

\section{Acknowledgments}

Many thanks to all of the teammates who helped with this research.

\section{References}

1. Virani SS, Alonso A, Benjamin EJ, Bittencourt MS, Callaway CW, Carson AP, Chamberlain AM, Chang AR, Cheng S, Delling FN et al: Heart Disease and Stroke Statistics-2020 Update: A Report From the American Heart Association. Circulation 2020, 141(9): e139-e596.

2. Jin Y, Wu L, Tang Y, Cao Y, Li S, Shen J, Yue S, Qu C, Shan C, Cui X et al: UFLC-Q-TOF/MS based screening and identification of the metabolites in plasma, bile, urine and feces of normal and blood stasis rats after oral administration of hydroxysafflor yellow A. Journal of chromatography B, Analytical technologies in the biomedical and life sciences 2016, 1012-1013:124-129.

3. Li L, Dong P, Hou C, Cao F, Sun S, He F, Song Y, Li S, Bai Y, Zhu D: Hydroxysafflor yellow A (HSYA) attenuates hypoxic pulmonary arterial remodelling and reverses right ventricular hypertrophy in rats. J Ethnopharmacol 2016, 186:224-233.

4. Tien YC, Lin JY, Lai CH, Kuo CH, Lin WY, Tsai CH, Tsai FJ, Cheng YC, Peng WH, Huang CY: Carthamus tinctorius L. prevents LPS-induced TNFalpha signaling activation and cell apoptosis through JNK1/2-NFkappaB pathway inhibition in H9c2 cardiomyoblast cells. J Ethnopharmacol 2010, 130(3):505-513. 
5. Zhou D, Qu Z, Wang H, Su Y, Wang Y, Zhang W, Wang Z, Xu Q: The effect of hydroxy safflower yellow A on coronary heart disease through Bcl-2/Bax and PPAR-y. Experimental and therapeutic medicine 2018, 15(1):520-526.

6. Zhou MX, Fu JH, Zhang Q, Wang JQ: Effect of hydroxy safflower yellow A on myocardial apoptosis after acute myocardial infarction in rats. Genetics and molecular research: GMR 2015, 14(2):31333141.

7. Liao H, Li Y, Zhai X, Zheng B, Banbury L, Zhao X, Li R: Comparison of Inhibitory Effects of Safflower Decoction and Safflower Injection on Protein and mRNA Expressions of iNOS and IL-1 $\beta$ in LPSActivated RAW264.7 Cells. Journal of immunology research 2019, 2019:1018274.

8. Zhang RZ, Yu SJ, Bai H, Ning K: TCM-Mesh: The database and analytical system for network pharmacology analysis for TCM preparations. Scientific reports 2017, 7(1):2821.

9. Zhang J, Liang R, Wang L, Yang B: Effects and mechanisms of Danshen-Shanzha herb-pair for atherosclerosis treatment using network pharmacology and experimental pharmacology. J Ethnopharmacol 2019, 229:104-114.

10. Cui S, Chen S, Wu Q, Chen T, Li S: A network pharmacology approach to investigate the antiinflammatory mechanism of effective ingredients from Salvia miltiorrhiza. International immunopharmacology 2020, 81:106040.

11. Wang Y, Shi Y, Zou J, Zhang X, Liang Y, Tai J, Cui C, Wang M, Guo D: Network pharmacology exploration reveals a common mechanism in the treatment of cardio-cerebrovascular disease with Salvia miltiorrhiza Burge. and Carthamus tinctorius L. BMC complementary medicine and therapies 2020, 20(1):351.

12. Vivancos M, Moreno JJ: beta-Sitosterol modulates antioxidant enzyme response in RAW 264.7 macrophages. Free radical biology \& medicine 2005, 39(1):91-97.

13. Chen T, Zhang X, Zhu G, Liu H, Chen J, Wang Y, He X: Quercetin inhibits TNF-a induced HUVECs apoptosis and inflammation via downregulating NF-kB and AP-1 signaling pathway in vitro. Medicine 2020, 99(38):e22241.

14. Che J, Liang B, Zhang Y, Wang Y, Tang J, Shi G: Kaempferol alleviates ox-LDL-induced apoptosis by up-regulation of autophagy via inhibiting PI3K/Akt/mTOR pathway in human endothelial cells. Cardiovascular pathology: the official journal of the Society for Cardiovascular Pathology 2017, $31: 57-62$.

15. Feng Z, Wang C, Yue, Jin, Meng Q, Wu J, Sun H: Kaempferol-induced GPER upregulation attenuates atherosclerosis via the PI3K/AKT/Nrf2 pathway. Pharmaceutical biology 2021, 59(1):1106-1116.

16. Zhang X, Qin Y, Ruan W, Wan X, Lv C, He L, Lu L, Guo X: Targeting inflammation-associated AMPK/Mfn-2/MAPKs signaling pathways by baicalein exerts anti-atherosclerotic action. Phytotherapy research: PTR 2021, 35(8):4442-4455.

17. Tucka J, Yu H, Gray K, Figg N, Maguire J, Lam B, Bennett M, Littlewood T: Akt1 regulates vascular smooth muscle cell apoptosis through FoxO3a and Apaf1 and protects against arterial remodeling and atherosclerosis. Arteriosclerosis, thrombosis, and vascular biology 2014, 34(11):2421-2428. 
18. Song L, Yang H, Wang HX, Tian C, Liu Y, Zeng XJ, Gao E, Kang YM, Du J, Li HH: Inhibition of 12/15 lipoxygenase by baicalein reduces myocardial ischemia/reperfusion injury via modulation of multiple signaling pathways. Apoptosis: an international journal on programmed cell death 2014 , 19(4):567-580.

19. Lin J, Jiang J, Zhou R, Li X, Ye J: MicroRNA-451b Participates in Coronary Heart Disease By Targeting VEGFA. Open medicine (Warsaw, Poland) 2018, 15:1-7.

20. Yang Y, Mao W, Wang L, Lu L, Pang Y: Circular RNA circLMF1 regulates PDGF-BB-induced proliferation and migration of human aortic smooth muscle cells by regulating the miR-125a3p/VEGFA or FGF1 axis. Clinical hemorheology and microcirculation 2021.

21. Harjes U, Bridges E, Mclntyre A, Fielding BA, Harris AL: Fatty acid-binding protein 4, a point of convergence for angiogenic and metabolic signaling pathways in endothelial cells. The Journal of biological chemistry 2014, 289(33):23168-23176.

22. Wang G, Liu X, Li X, Zhao Y: Suppression of PAPP-A mitigates atherosclerosis by mediating macrophage polarization via STAT3 signaling. Biochemical and biophysical research communications 2021, 543:29-37.

23. Ng IH, Yeap YY, Ong LS, Jans DA, Bogoyevitch MA: Oxidative stress impairs multiple regulatory events to drive persistent cytokine-stimulated STAT3 phosphorylation. Biochimica et biophysica acta 2014, 1843(3):483-494.

24. Wei Z, Jiang W, Wang H, Li H, Tang B, Liu B, Jiang H, Sun X: The IL-6/STAT3 pathway regulates adhesion molecules and cytoskeleton of endothelial cells in thromboangiitis obliterans. Cellular signalling 2018, 44:118-126.

25. Vasamsetti SB, Karnewar S, Kanugula AK, Thatipalli AR, Kumar JM, Kotamraju S: Metformin inhibits monocyte-to-macrophage differentiation via AMPK-mediated inhibition of STAT3 activation: potential role in atherosclerosis. Diabetes 2015, 64(6):2028-2041.

26. Liao XH, Wang N, Zhao DW, Zheng DL, Zheng L, Xing WJ, Ma WJ, Bao LY, Dong J, Zhang TC: STAT3 Protein Regulates Vascular Smooth Muscle Cell Phenotypic Switch by Interaction with Myocardin. The Journal of biological chemistry 2015, 290(32):19641-19652.

27. Yu HM, Zhi JL, Cui Y, Tang EH, Sun SN, Feng JQ, Chen PX: Role of the JAK-STAT pathway in protection of hydrogen peroxide preconditioning against apoptosis induced by oxidative stress in PC12 cells. Apoptosis: an international journal on programmed cell death 2006, 11(6):931-941.

28. Ma L, Zhang L, Wang B, Wei J, Liu J, Zhang L: Berberine inhibits Chlamydia pneumoniae infectioninduced vascular smooth muscle cell migration through downregulating MMP3 and MMP9 via PI3K. European journal of pharmacology 2015, 755:102-109.

29. Deleon-Pennell KY, Altara R, Yabluchanskiy A, Modesti A, Lindsey ML: The circular relationship between matrix metalloproteinase-9 and inflammation following myocardial infarction. IUBMB life 2015, 67(8):611-618.

30. Eilenberg W, Stojkovic S, Kaider A, Kozakowski N, Domenig CM, Burghuber C, Nanobachvili J, Huber K, Klinger M, Neumayer $C$ et al: NGAL and MMP-9/NGAL as biomarkers of plaque vulnerability and 
targets of statins in patients with carotid atherosclerosis. Clinical chemistry and laboratory medicine 2017, 56(1):147-156.

31. Hernández-Aguilera A, Fibla M, Cabré N, Luciano-Mateo F, Camps J, Fernández-Arroyo S, MartínParedero V, Menendez JA, Sirvent JJ, Joven J: Chemokine (C-C motif) ligand 2 and coronary artery disease: Tissue expression of functional and atypical receptors. Cytokine 2020, 126:154923.

32. Qin Y, Mao W, Pan L, Sun Y, Fan F, Zhao Y, Cui Y, Wei X, Kohama K, Li F et al: Inhibitory effect of recombinant human CXCL8(3-72) K11R/G31P on atherosclerotic plaques in a mouse model of atherosclerosis. Immunopharmacology and immunotoxicology 2019, 41(3):446-454.

33. Bowman JD, Surani S, Horseman MA: Endotoxin, Toll-like Receptor-4, and Atherosclerotic Heart Disease. Current cardiology reviews 2017, 13(2):86-93.

34. Morange PE, Tiret L, Saut N, Luc G, Arveiler D, Ferrieres J, Amouyel P, Evans A, Ducimetiere P, Cambien F et al: TLR4/Asp299Gly, CD14/C-260T, plasma levels of the soluble receptor CD14 and the risk of coronary heart disease: The PRIME Study. European journal of human genetics: EJHG 2004, 12(12):1041-1049.

35. Heiskanen $M$, Kähönen $M$, Hurme $M$, Lehtimäki T, Mononen $N$, Juonala $M$, Hutri-Kähönen N, Viikari J, Raitakari O, Hulkkonen J: Polymorphism in the IL10 promoter region and early markers of atherosclerosis: the Cardiovascular Risk in Young Finns Study. Atherosclerosis 2010, 208(1):190196.

36. Khyzha N, Khor M, DiStefano PV, Wang L, Matic L, Hedin U, Wilson MD, Maegdefessel L, Fish JE: Regulation of CCL2 expression in human vascular endothelial cells by a neighboring divergently transcribed long noncoding RNA. Proceedings of the National Academy of Sciences of the United States of America 2019, 116(33):16410-16419.

37. Yu Z, Tan BK, Dainty S, Mattey DL, Davies SJ: Hypoalbuminaemia, systemic albumin leak and endothelial dysfunction in peritoneal dialysis patients. Nephrology, dialysis, transplantation: official publication of the European Dialysis and Transplant Association - European Renal Association 2012, 27(12):4437-4445.

38. Jain T, Nikolopoulou EA, Xu Q, Qu A: Hypoxia inducible factor as a therapeutic target for atherosclerosis. Pharmacology \& therapeutics 2018, 183:22-33.

39. Tawakol A, Singh P, Mojena M, Pimentel-Santillana M, Emami H, MacNabb M, Rudd JH, Narula J, Enriquez JA, Través PG et al: HIF-1 $a$ and PFKFB3 Mediate a Tight Relationship Between Proinflammatory Activation and Anerobic Metabolism in Atherosclerotic Macrophages. Arteriosclerosis, thrombosis, and vascular biology 2015, 35(6):1463-1471.

40. Yan H, Ma Y, Li Y, Zheng X, Lv P, Zhang Y, Li J, Ma M, Zhang L, Li C et al: Insulin inhibits inflammation and promotes atherosclerotic plaque stability via PI3K-Akt pathway activation. Immunology letters $2016,170: 7-14$.

41. Li T, Li D, Xu H, Zhang H, Tang D, Cao H: Wen-Xin Decoction ameliorates vascular endothelium dysfunction via the PI3K/AKT/eNOS pathway in experimental atherosclerosis in rats. BMC complementary and alternative medicine 2016, 16:27. 
42. Jian D, Wang Y, Jian L, Tang H, Rao L, Chen K, Jia Z, Zhang W, Liu Y, Chen X et al: METTL14 aggravates endothelial inflammation and atherosclerosis by increasing FOXO1 N6-methyladeosine modifications. Theranostics 2020, 10(20):8939-8956.

43. Babaee M, Chamani E, Ahmadi R, Bahreini E, Balouchnejadmojarad T, Nahrkhalaji AS, Fallah S: The expression levels of miRNAs-27a and 23a in the peripheral blood mononuclear cells (PBMCs) and their correlation with FOXO1 and some inflammatory and anti-inflammatory cytokines in the patients with coronary artery disease (CAD). Life sciences 2020, 256:117898.

44. Chen L, Gao B, Zhang Y, Lu H, Li X, Pan L, Yin L, Zhi X: PAR2 promotes M1 macrophage polarization and inflammation via FOXO1 pathway. Journal of cellular biochemistry 2019, 120(6):9799-9809.

45. Puthanveetil P, Wan A, Rodrigues B: FoxO1 is crucial for sustaining cardiomyocyte metabolism and cell survival. Cardiovascular research 2013, 97(3):393-403.

46. Potente M, Urbich C, Sasaki K, Hofmann WK, Heeschen C, Aicher A, Kollipara R, DePinho RA, Zeiher $A M$, Dimmeler S: Involvement of Foxo transcription factors in angiogenesis and postnatal neovascularization. The Journal of clinical investigation 2005, 115(9):2382-2392.

47. Chong ZZ, Wang S, Shang YC, Maiese K: Targeting cardiovascular disease with novel SIRT1 pathways. Future cardiology 2012, 8(1):89-100.

48. Tsuchiya K, Tanaka J, Shuiqing Y, Welch CL, DePinho RA, Tabas I, Tall AR, Goldberg IJ, Accili D: FoxOs integrate pleiotropic actions of insulin in vascular endothelium to protect mice from atherosclerosis. Cell metabolism 2012, 15(3):372-381.

49. Kitada M, Ogura Y, Koya D: The protective role of Sirt1 in vascular tissue: its relationship to vascular aging and atherosclerosis. Aging 2016, 8(10):2290-2307.

50. Wu Q, Hu Y, Jiang M, Wang F, Gong G: Effect of Autophagy Regulated by Sirt1/FoxO1 Pathway on the Release of Factors Promoting Thrombosis from Vascular Endothelial Cells. International journal of molecular sciences 2019, 20(17).

51. Dong Y, Chen H, Gao J, Liu Y, Li J, Wang J: Molecular machinery and interplay of apoptosis and autophagy in coronary heart disease. Journal of molecular and cellular cardiology 2019, 136:27-41.

52. Edlich F: BCL-2 proteins and apoptosis: Recent insights and unknowns. Biochemical and biophysical research communications 2018, 500(1):26-34.

53. Zhu Z, Li J, Zhang X: Salidroside protects against ox-LDL-induced endothelial injury by enhancing autophagy mediated by SIRT1-Fox01 pathway. BMC complementary and alternative medicine 2019, 19(1):111.

54. Menghini R, Casagrande V, Iuliani G, Rizza S, Mavilio M, Cardellini M, Federici M: Metabolic aspects of cardiovascular diseases: Is FoxO1 a player or a target? The international journal of biochemistry \& cell biology 2020, 118:105659.

\section{Tables}

Due to technical limitations, tables are only available as a download in the Supplemental Files section. 
Figures

\section{Figure 1}

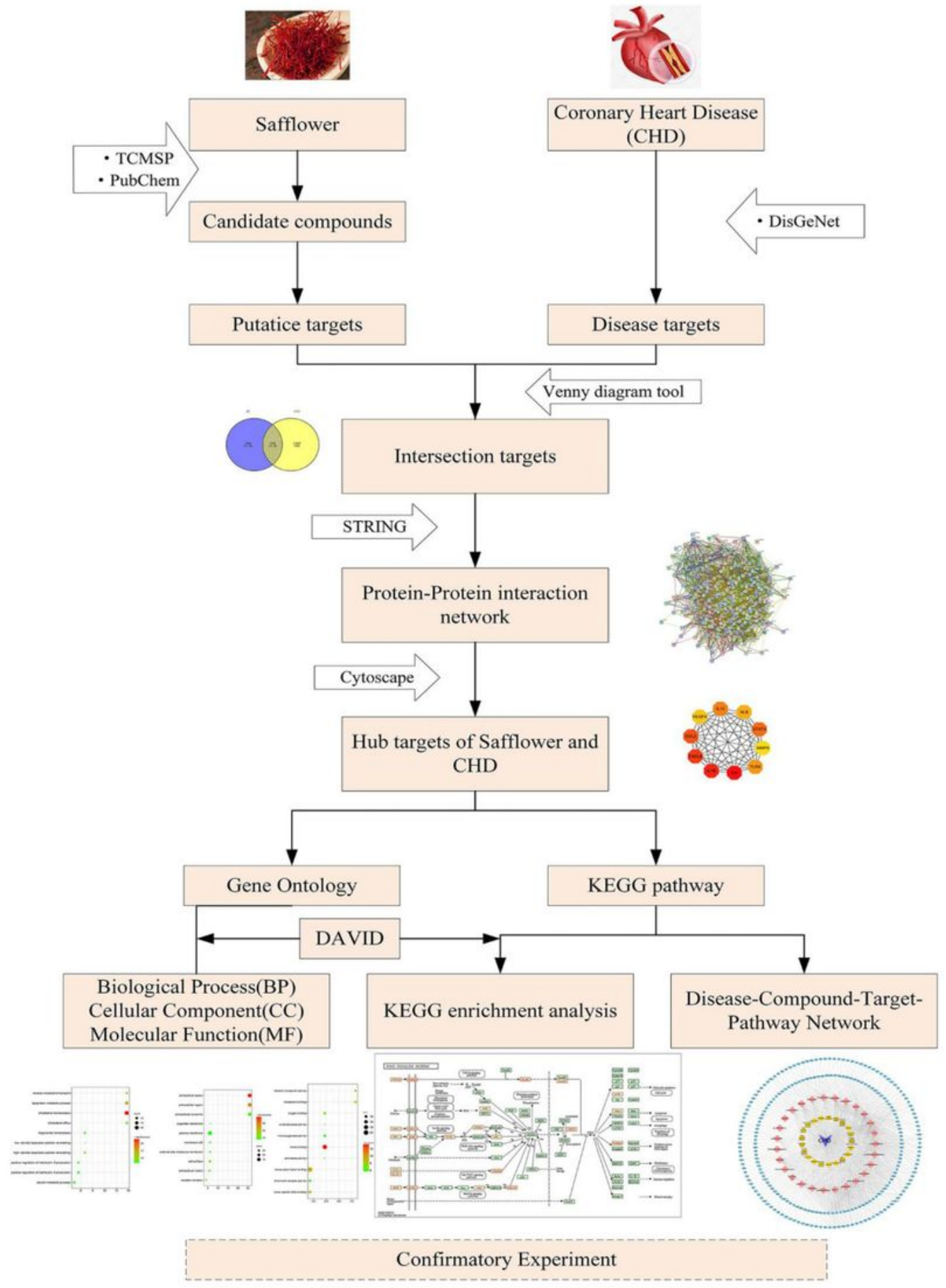

Figure 1

The overall process flow of the study. 


\section{Figure 2}

Safflower

CHD

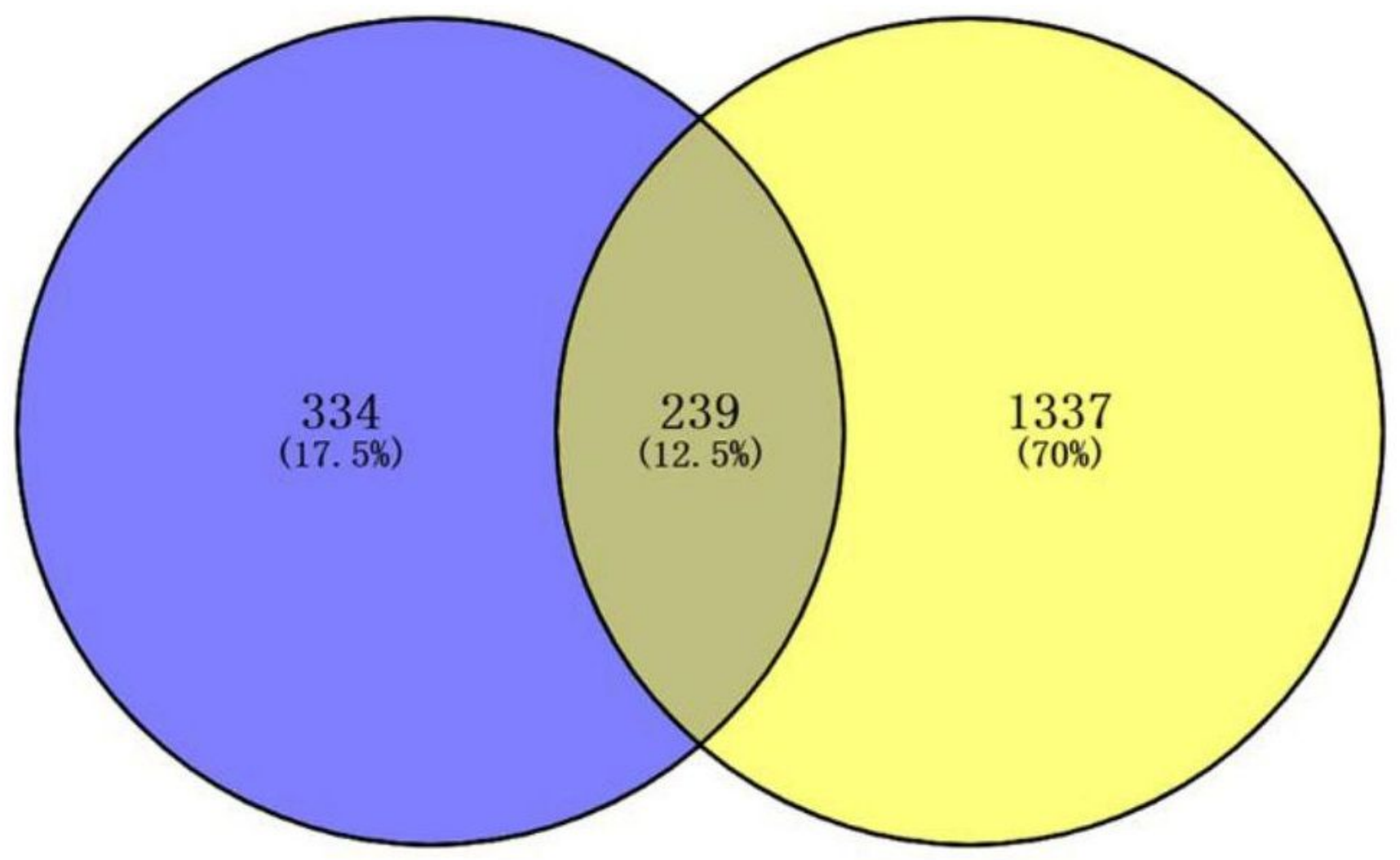

Figure 2

Venn diagram of safflower and CHD-related targets. The color purple represents the number of safflower active ingredient targets, the color yellow represents the number of coronary heart disease targets, and the middle section represents the cross-targets of both. 


\section{Figure 3}



\section{Figure 3}

Protein-protein interaction network. These loop nodes represent interacting proteins and the edges represent interactions between proteins. 
Figure 4

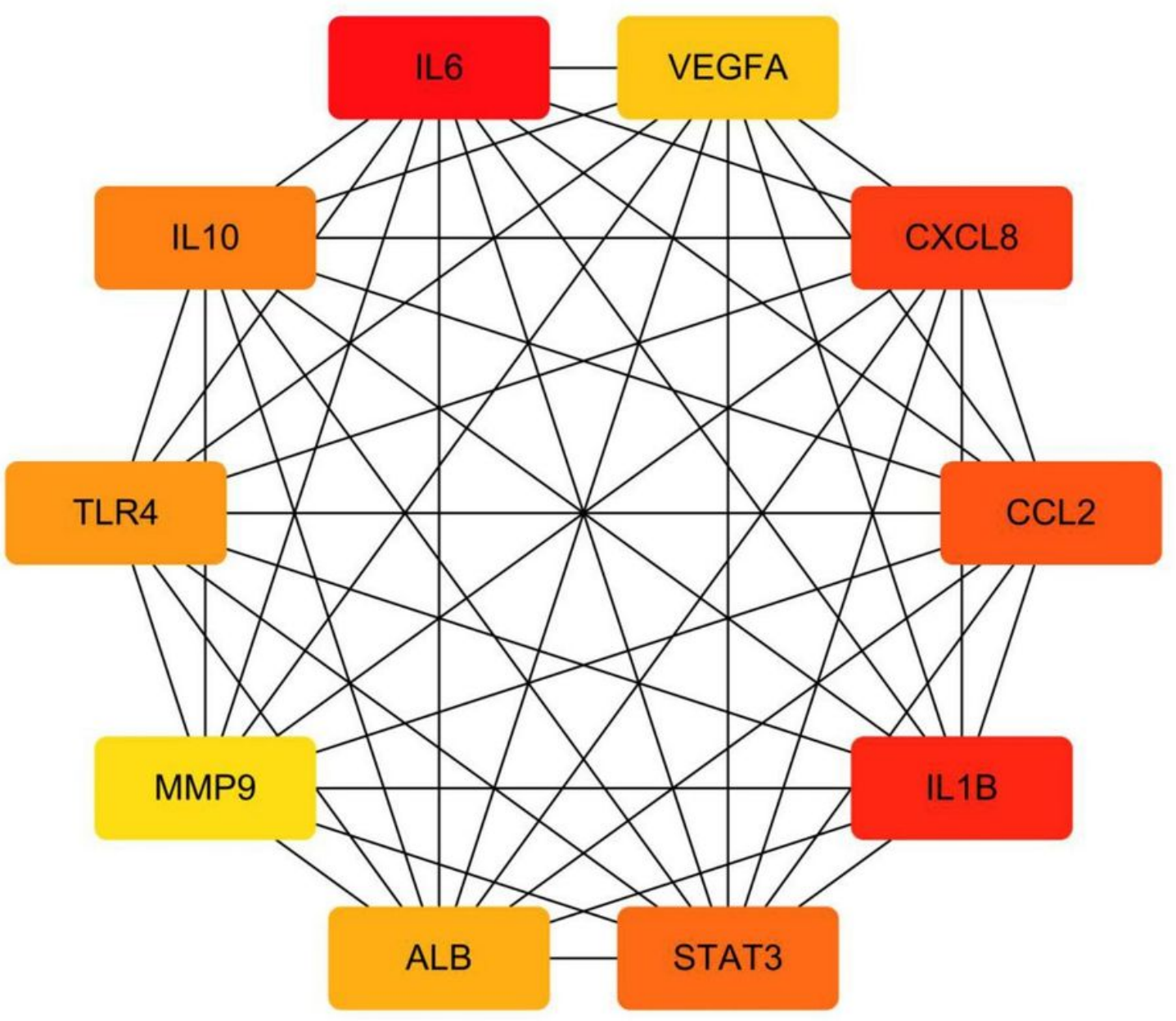

Figure 4

MCC algorithm analysis of the top 10 safflower hub gene networks for CHD therapy. 


\section{Figure 5}

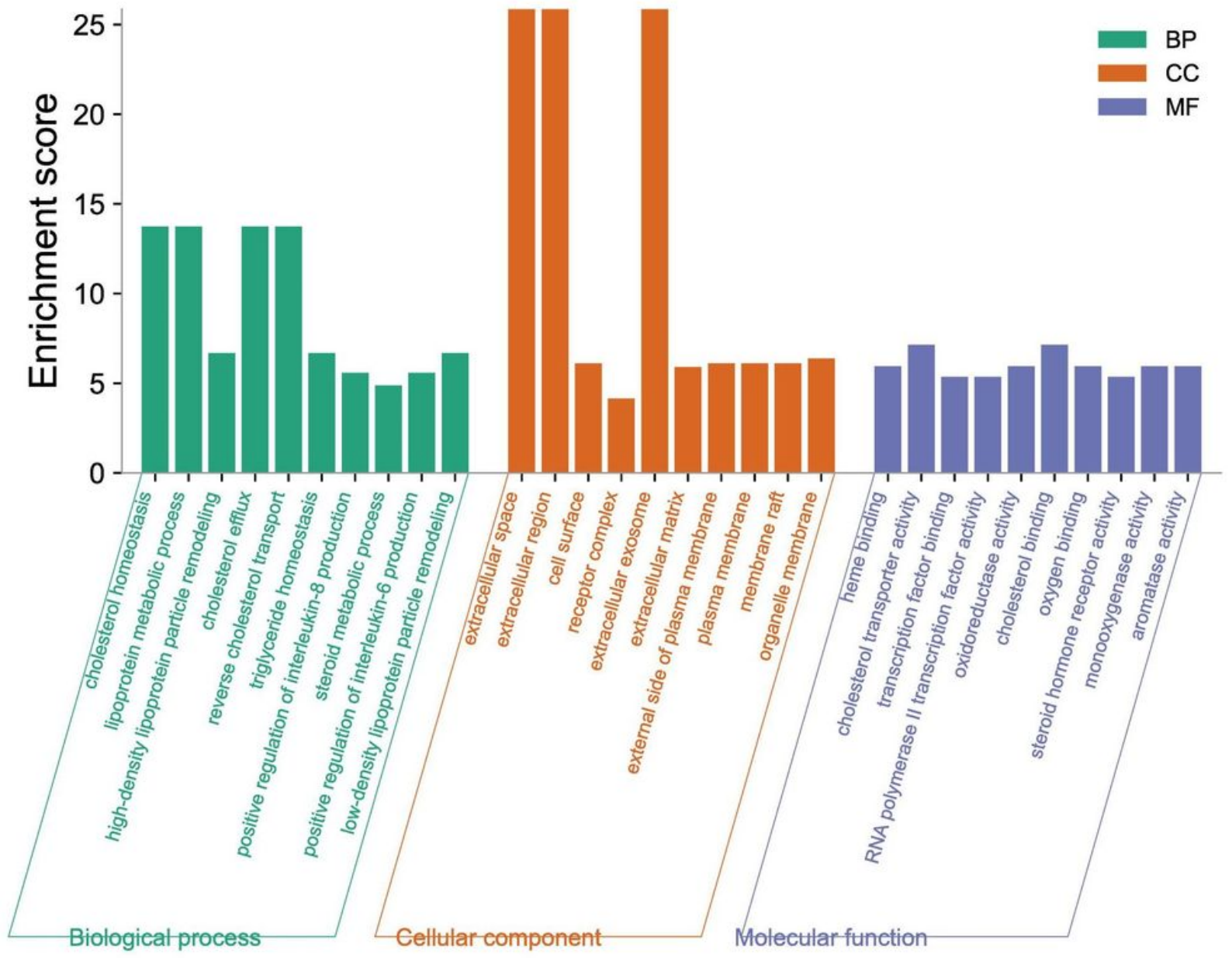

Figure 5

GO function enrichment chart of safflower in the treatment of CHD. Green denotes biological processes, orange denotes cellular components, and purple denotes molecular function. 


\section{Figure 6}

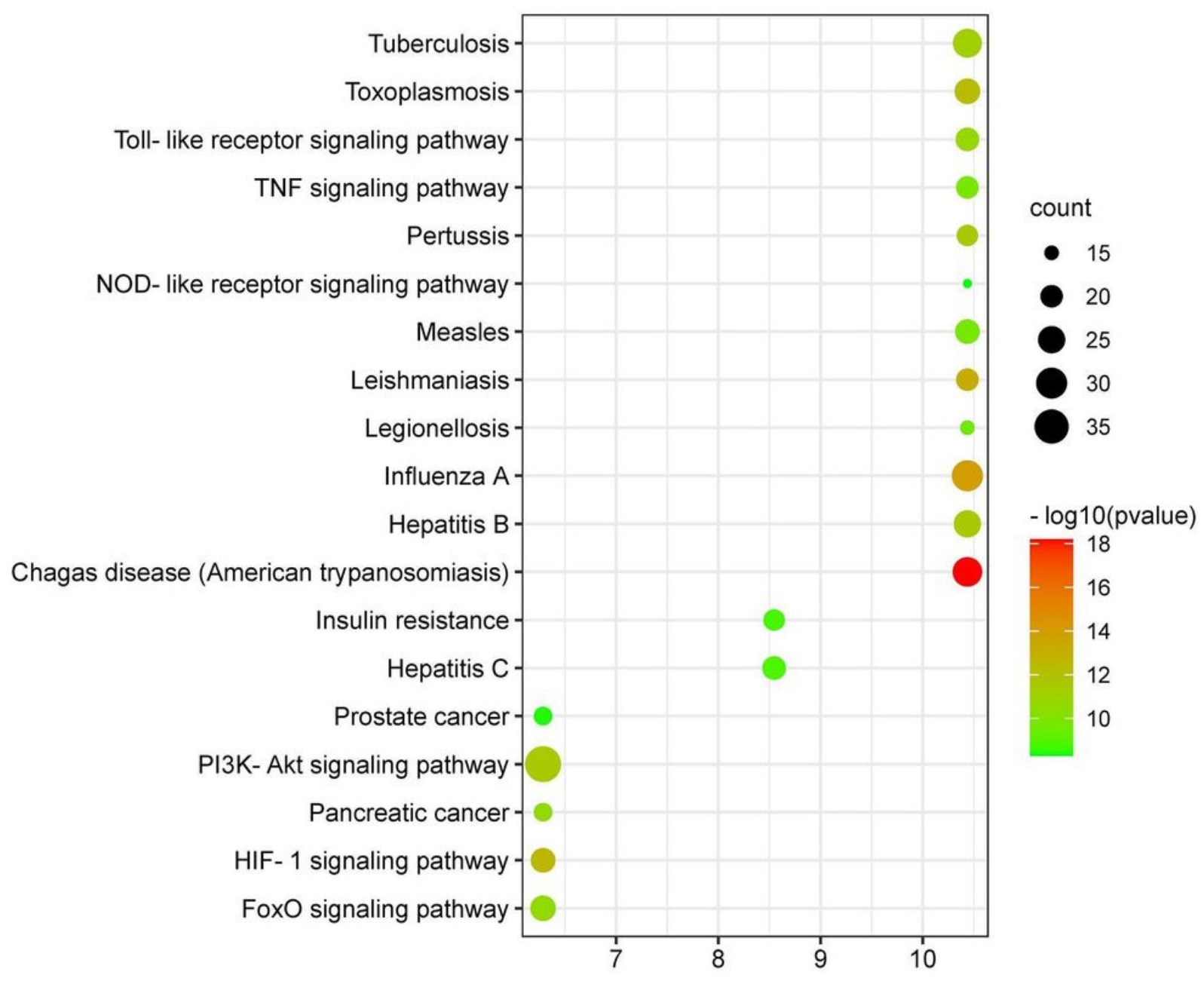

\section{Figure 6}

KEGG pathway analyses for the molecular signal pathway of safflower in the treatment of CHD.The node size represents the number of target genes enriched, and the node color from red to green represents the $P$ value from large to small. 
Figure 7

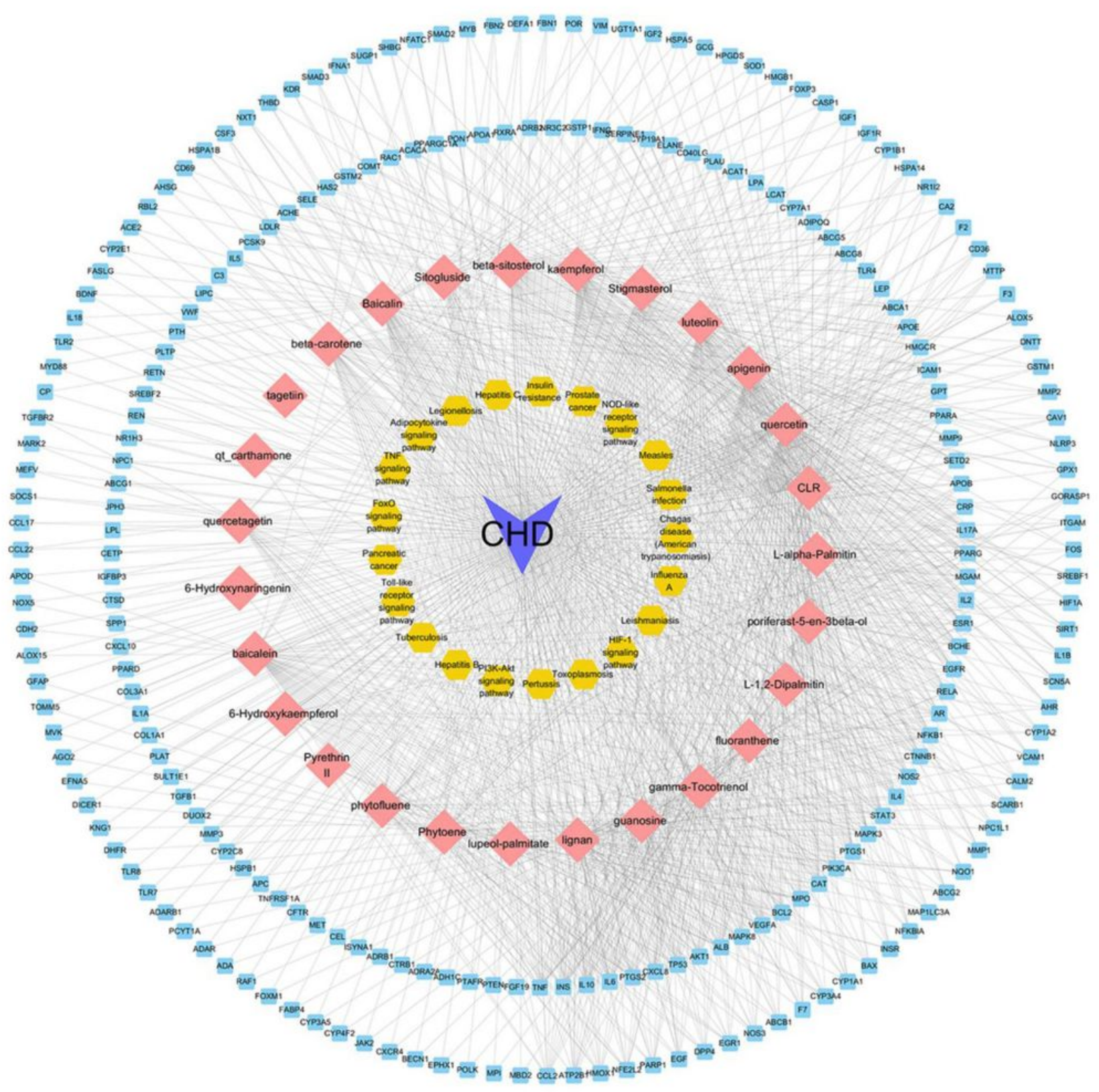

Figure 7

A Disease-Compound-Target-Pathway network was structured. Purple triangles symbolize CHD, pink rhombuses represent safflower chemical compounds related to common targets, blue squares represent chemical compound and CHD targets, while yellow hexagons highlight key biological pathways. 


\section{Figure 8}

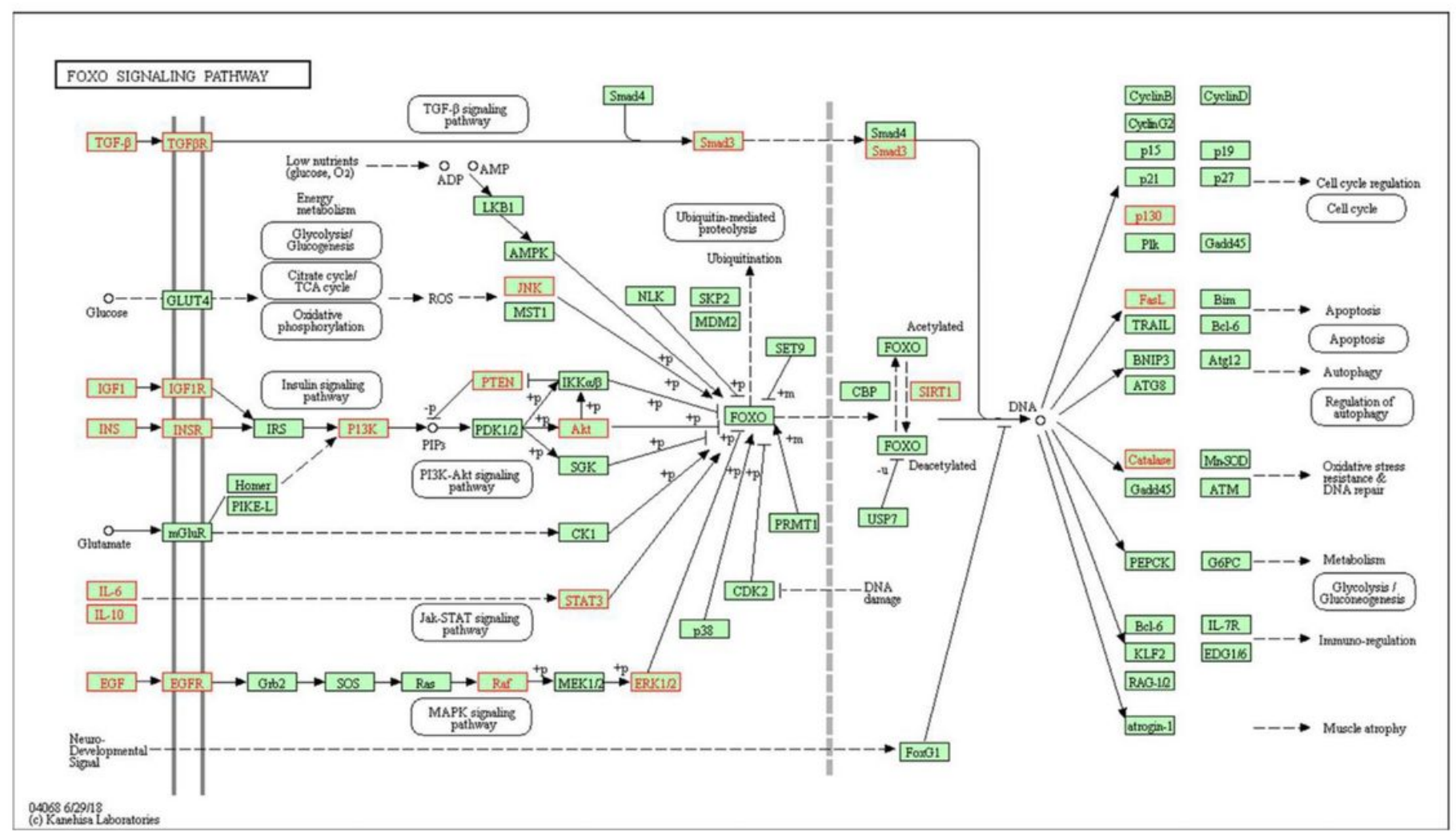

\section{Figure 8}

FoxO signaling pathway. The targets associated with the core component-target-pathway network are represented by red rectangles. 
Figure 9

A

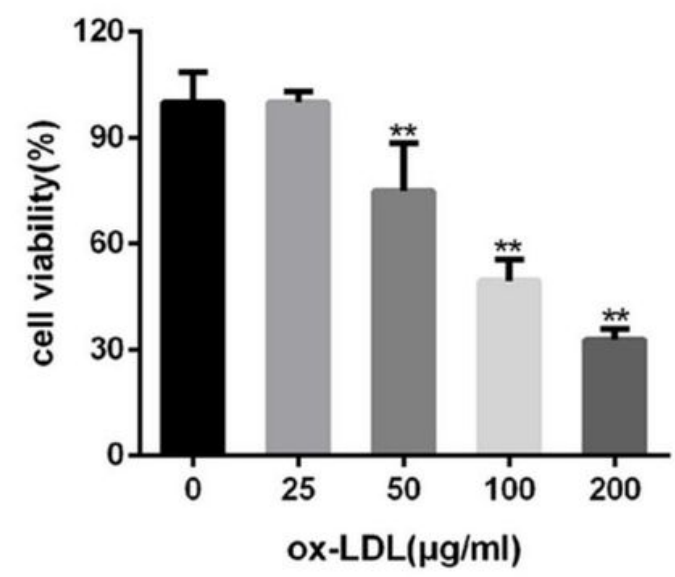

B

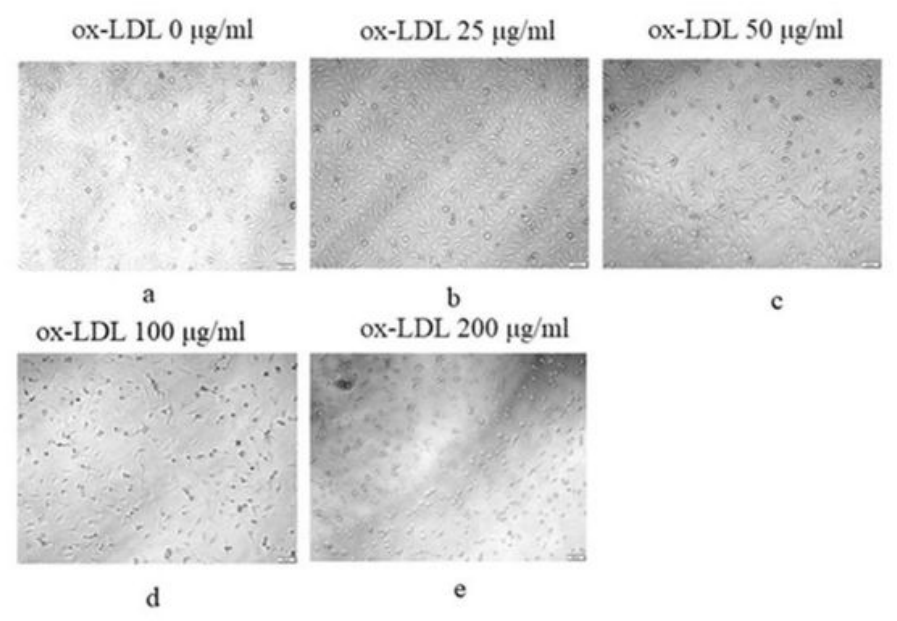

Figure 9

A. The cell survival rate of EAhy926 cells under different concentrations of ox-LDL. ( $* \star P<0.01$ vs. ox-LDL $0 \mu \mathrm{g} / \mathrm{ml}$ group). B. Cell morphology of EAhy 926 cells under different concentrations of ox-LDL (x10).

Figure 10

A

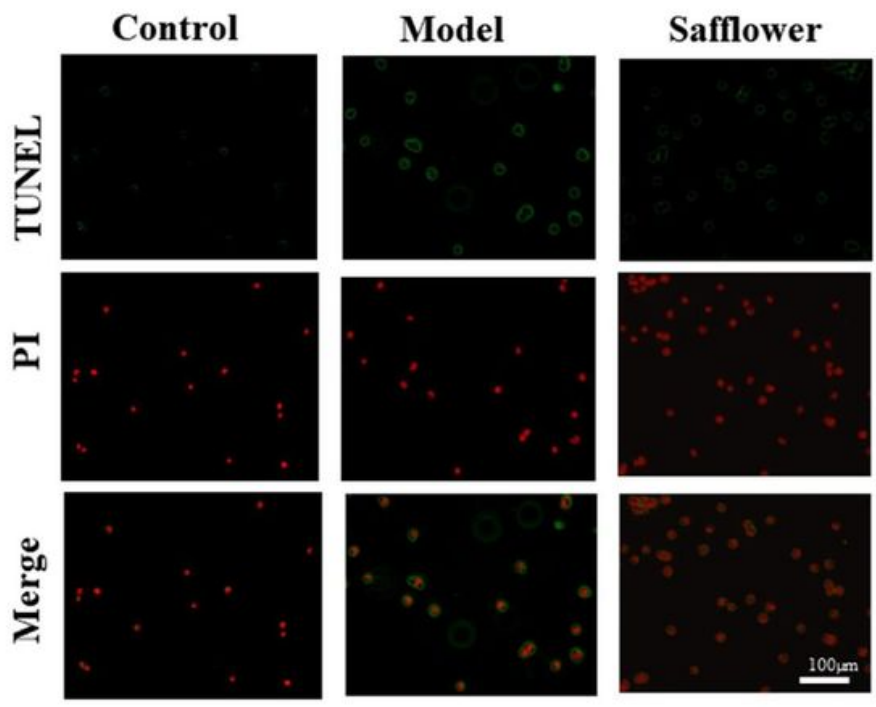

B

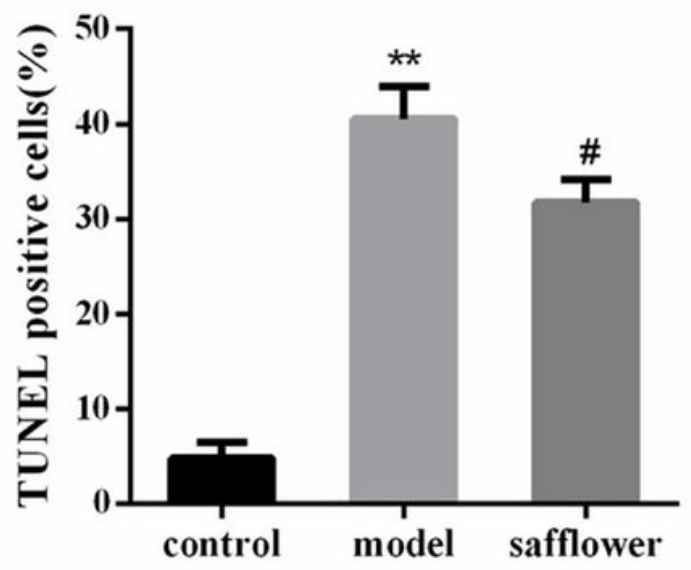


Figure 10

Safflower inhibits apoptosis of EAhy926 cells caused by ox-LDL. (A). TUNEL dye was applied to cells, which were then observed under a fluorescence microscope (x 20). (B). Quantitative analysis of TUNEL positive rates. ( ${ }^{\star *} P<0.01$ vs. control group. ${ }^{\#} P<0.05$ vs. model group; $\mathrm{n}=3$ independent cell culture preparations).

Figure 11

$\mathbf{A}$

B

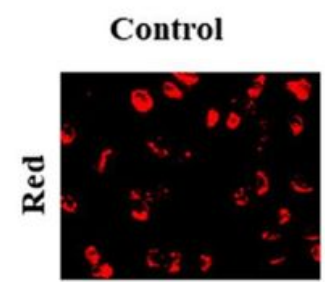

Model

Safflower
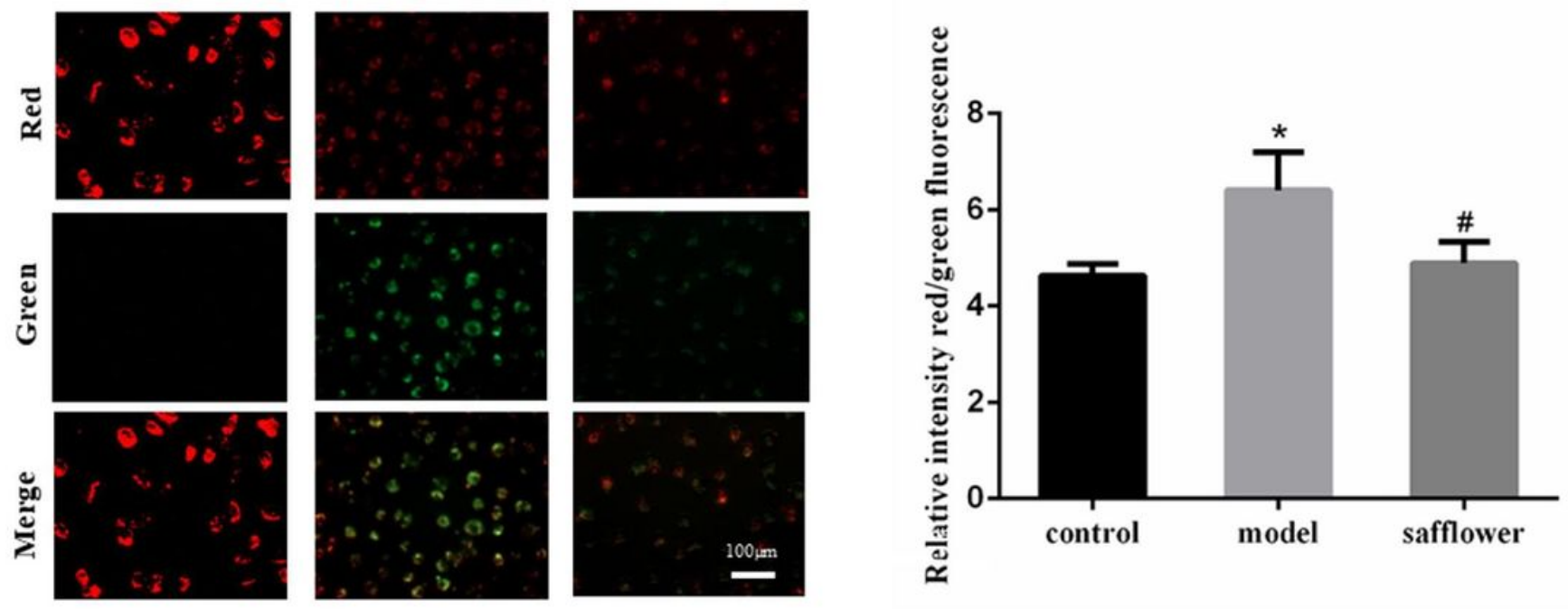

\section{Figure 11}

Effects of safflower on the mitochondrial membrane potential of ox-LDL induced EAhy926 cells. (A). JC-1 was used to stain EAhy 926 cells, which were then observed under a fluorescent microscope (x 20). (B). Quantitative analysis of the red/green rates after JC-1 staining; ${ }^{*} P<0.05$ vs. control group. ${ }^{\#} P<0.05$ vs. model group. $\mathrm{n}=3$ independent cell culture preparations. 
Figure 12

a

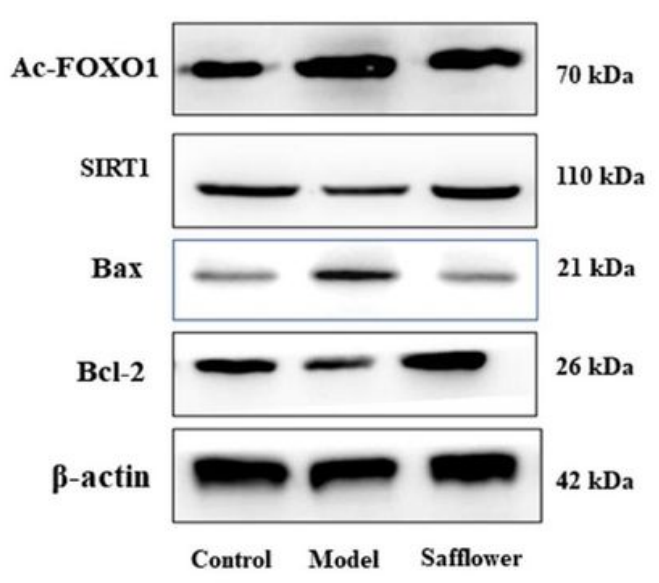

$\mathrm{b}$

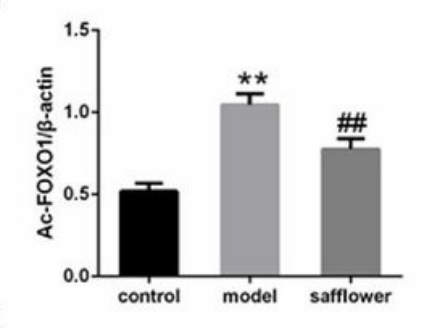

d

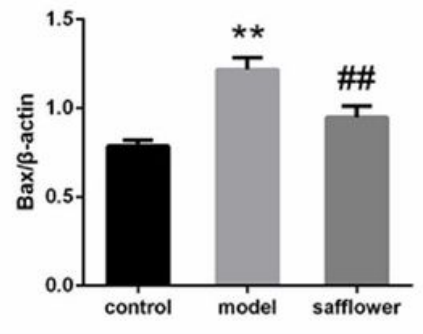

$\mathrm{C}$

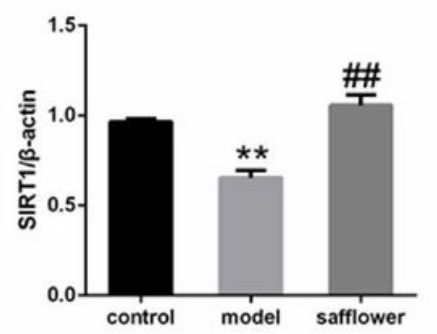

$\mathrm{e}$

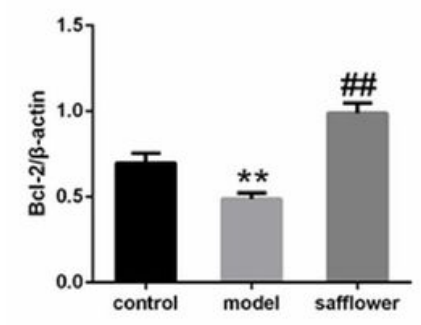

Figure 12

Effects of safflower on the expression of Ac-Fox01, STIR1, and Bax, Bcl-2 in ox-LDL induced EAhy926 cells. Expression levels of Ac-Fox01, STIR1, Bax, and Bcl-2 were detected by western blot analysis. ( $* \star P<$ 0.01 vs. control group; ${ }^{\# \#} P<0.01$ vs. model group).

\section{Supplementary Files}

This is a list of supplementary files associated with this preprint. Click to download.

- safflowertableBMC2.pdf 